\title{
ON THE EMPLOYMENT
}

\section{or \\ THE HEAT OF ELECTRICITY \\ IN PRACTICAL SURGERY.}

\author{
BY \\ JOHN MARSHALL, F.R.C.S. \\ ASSISTANT-SURGEON TO THE UNIVERSITY COLLEGE HOSPITAL. \\ COMRUNICATED BX \\ RICHARD QUAIN, ESQ., F.R.S. \\ Received April 7th.-Read April 22d, 1851.
}

THe object of the present paper being to direct the attention of the Profession to the employment of the Heat of Electricity in Practical Surgery, I will, in the first place, describe the case, by the peculiarities of which its application was originally suggested.

Early in September last, (1850,) Richard W. H-, a native of London, aged 25, of delicate constitution and strumous habit, who had chiefly resided in the metropolis, and had been for some years engaged therein as a linendraper's shopman, came under my care, as an out-patient, at the University College Hospital, with an obstinate fistula in the right cheek, resulting from a succession of abscesses. According to the statement which he made, it appears that in November, 1849, (being then out of health,) he was first troubled with a swelling of the cheek, the cause of which he does not know. For a period of nine or ten weeks, the swelling became alternately better and worse-being some- 
times, after the use of fomentations and purgatives, reduced to a small lump the size of a bean, - and at other times, enlarging even in the course of a couple of hours, so that the mouth could hardly be opened. Up to the beginning of February, this swelling had caused more inconvenience than pain; but the cheek then became so swollen, stiff, and painful, that he applied to Mr. Ancell, who discovered a small deep-seated abscess, which after three or four days, was opened by an incision inside the cheek. During the next four weeks, the discharge continued, and it became necessary, owing to further suppuration in the neighbourhood, to make other incisions, and ultimately to unite them on the inner surface of the cheek. About the middle of March, the cheek again swelled, so as nearly to close the right eye, and a fresh formation of pus, accompanied by constitutional disturbance, took place, - on this occasion tending towards the skin, which became much inflamed. Two separate punctures through the integument, made at different times, were found necessary to evacuate the accumulated pus, and soon after a large probe could be passed quite through the cheek, the passage having two small external orifices and one long internal opening. Finally it became necessary to lay open the two outer orifices into one.

By the use of poultices, lotions for the mouth, occasional purgatives, and a course of tonic medicine, the suppurating cavities in the cheek gradually diminished, and, together with the external and internal wounds, became so contracted, that there remained merely a narrow fistulous passage leading into the mouth. Two or three times, in the early part of May, the external orifice of the fistula temporarily healed, but the cicatrix always became again inflamed, and was burst through by matter accumulating behind it; while the discharge from the inner opening never entirely ceased. About the beginning of June, as the fistulous passage did not heal, it was thought advisable to try the effect of improving the general health, and Mr. H- was sent to Margate, where he attended, as an out-patient, at "The Royal Seabathing Infirmary," under the care of Mr. Field. Here, 
besides proper medical treatment, he took sea-baths, an Iodine ointment was applied to the face, and an Iodine injection made use of to stimulate the walls of the fistula. Once or twice, the outer wound again closed up, but only for two or three days. At the end of six weeks he left Margate, very much more vigorous in health, but (understanding from Mr. Field that probably even a six months' residence there would not suffice to cure the fistula) he returned to London, and very soon after, an increased discharge escaped from both ends of the fistulous passage, which was pronounced by $\mathrm{Mr}$. Ancell to be as intractable as ever. It continued in the same state until the $3 \mathrm{~d}$ of September, when I first saw the patient at the Hospital.

At that date, I found on the right cheek, behind the angle of the month, a puckered depression, at the bottom of which was an elevated and inflamed cicatrix, about an inch long and a quarter of an inch wide; on the fore-part of the cicatrix a small fungous granulation marked the principal orifice of the fistula. About half an inch behind this was a smaller orifice, apparently quite recently ulcerated, which led into the side of the fistulous track. On the inside of the cheek, a long cleft extended backwards on a level with the lower molar teeth. At the further part of this cleft, opposite the second molar tooth, a fleshy projection indicated the internal fistulous orifice. The direction of the fistula was at first obliquely inwards through the skin, then backwards, and then inwards again into the mouth; its length was $1 \frac{3}{4}$ inches; it would only just admit a fine probe, and was somewhat narrowed at either orifice. The tissues around it were hard, but not tender on pressure. A small quantity of a very thick whitish, almost curdy pus, escaped from both ends of it. Its position was altogether below the Stenonian duct, and no saliva ever flowed through it. The patient was at this time rather thinner than usual, and appeared anxious and miserable. The constant discharge and unsightly dressings annoyed himself and others, and rendered him objectionable, and even unfit for his situation as a shopman. 
Finding that the pus was somewhat pent up in the fistula, I enlarged the principal external orifice by about a quarter of an inch, and then, with a view of stimulating the lining membrane and maintaining a free exit for the matter, it was injected with a solution of Sulphate of Copper, and some threads of lint, moistened with the solution, were introduced into it. Under this treatment, in about three weeks, the hinder of the two external openings completely closed up, but the main fistulous track showed no disposition to heal. Other injections containing Sulphate of Zinc, Nitrate of Silver, or Iodine, and ultimately the solid caustic, were successively tried, being frequently used by myself, so as to ensure their efficient application. In the mean time, he took wine and porter, Quinine and Sulphuric Acid, and afterwards Quinine and Iron. His strength improved, the local stimulants employed produced increased action along the fistula; but even after six or seven weeks there was no disposition to adhesion of its sides.

After such persevering trials on the part of others as well as myself, it appeared probable that the treatment hitherto adopted would not succeed in closing a fistulous passage, the condition of which had undergone no material improvement for more than six months. The patient, moreover, becoming dispirited, it became necessary to try some other method, and I proposed to revive the inner surface of the fistula either by the actual cautery, or by dissecting out the callous tissue along it, and bringing the cut surface together by hare-lip pins and sutures. The former of these two methods seemed in every way preferable, but it was obviously difficult, or I might say impossible, effectually to cauterize, by the ordinary hot wire, the whole length of an extremely narrow and winding passage, nearly two inches long, through which a fine probe could only be insinuated by tedious manipulation; and cauterization of the accessible part only of the fistula, would probably have ended in no permanent advantage. It then occurred to me that a piece of platinum wire, which might be easily passed through the narrowest and most tortuous passage, might, by being made to form part of the 
circuit of a powerful galvanic battery, be so intensely heated throughout its whole length, while still lying in the fistula, as most effectually to cauterize every portion of its inner surface.

No rational objection appearing to this plan, I commenced by making some preliminary experiments on both dead and living animal tissues. In these trials, the objects held in view were :-

1st. To ascertain the possibility of obtaining a sufficiently equable cauterizing heat along the whole length of a wire in contact with a moist conducting substance, like animal tissue.

2dly. To determine the nature and extent of the effect produced on the tissue itself, and its consequences, both immediate and remote, in the living animal.

3dly. To learn by actual trial, before operating on the human subject, the size of wire best suited to accomplish the desired purpose, the battery power requisite to heat the wire under the peculiar circumstances, and the length of time during which the electric current should be allowed to traverse it.

4thly. To familiarise one's self with the practice of cauterization by this mode, so as to be able to adopt such contrivances and precautions as experience might suggest.

The experiments were made with a Grove's battery of four cells, each having a platina surface of thirty-two, and a zinc surface of thirty-eight square inches. Fine platina wire was the agent employed to localise the electric heat. On dead animal matter, it was found that a tubular portion of the tissue was destroyed immediately around the whole length of the imbedded portion of the wire, the thickness of tissue cauterized being somewhat greater at either end than towards the middle of the imbedded wire; the depth to which the cautery reached, depending on the power of the battery, the size of the wire, and the duration of the current, was, therefore, entirely under control. A few seconds only were required to destroy, effectually, the surfaces in actual contact with the heated wire. In the living animal, 
a rather longer time was needed to produce corresponding effects, but no mischief from laceration or bursting of the tissues, or from shock to the general system, arose. The opera tions were performed on a rabbit and dog, placed under the influence of chloroform, and consisted chiefly in the perforation of considerable thicknesses of the soft parts. Inflammation along the course of the cauterized track, separation of tubular sloughs thrown out at either orifice of the wound, and active granulation and suppuration, occurred in rapid succession, and finally the wounds, in all cases, quickly healed.

The practicability and safety of cauterization by the heat of electricity being thus established, I proceeded, on the 5th of November, in the presence of Professor Sharpey and Dr. Ditchfield, to submit my patient to the following operation. The same battery was employed as before. One of the poles was interrupted, the broken end terminating at a mercury cup; a fine platinum wire, $\frac{1}{50}$ th of an inch thick, was passed leisurely, and without producing pain, through the fistula, until it appeared in the mouth of the patient. The part of the wire outside the cheek was then twisted on to one of the stout copper poles of the battery, whilst the other end, visible at the inner orifice of the fistula, was brought into contact with the other pole, which, for that purpose, was passed into the mouth. During these arrangements, the circuit of the battery remained open. The patient, who had not taken chloroform, was now desired to keep quiet and allow his head to be firmly held. The galvanic circuit was then closed by dipping the interrupted pole into the mercury; when the platinum wire instantly became heated; and, at the expiration of nine seconds, the circuit was broken, the cauterization being supposed, from previous experience, to be, by that time, sufficiently complete. The poles of the battery being then removed, the platinum wire was found to cling slightly to the cauterized sides of the fistula, so that two fingers were held on the cheek whilst it was carefully withdrawn. Both orifices of the fistula would now have admitted a crow-quill, 
and were surrounded by a well-defined, opaque, whitish eschar. The patient expressed himself surprised at the small amount of pain produced by the operation; he had felt a sense of burning upon his cheek, and of pricking within the mouth; but no pain along the fistulous track. About five or ten minutes afterwards he went away, feeling nothing beyond a sense of stiffness in the cheek.

On the next two days, a little swelling and redness appeared along the course of the fistula, accompanied by the escape of a thin, brownish fluid. On the fourth day the sloughs visible at either opening were loosening; that at the inner orifice came away on the fifth day, that at the outer on the sixth day. The swelling of the surrounding parts aided in extruding the sloughs, and also in bringing together the sides of the fistula, the inner surface of which was now secreting healthy pus. The quantity of this secretion gradually diminished; on the eighth day the inner opening was no longer distinguishable; and on the 16th November, the eleventh day after the operation, the external opening was also soundly and permanently cicatrized.

About a week after this, a slight stiffness and swelling came on in the back part of the cheek ; and a small quantity of thick curdy pus, was again found escaping into the mouth from the deep cleft previously described on the inner side of the cheek. I thought the fistula had reopened; but it was not so. On examination I found a small opening, at the back part of this cleft, from which a single drop of pus could be squeezed by pressure over the middle of the masseter muscle; this opening led into a narrow blind pouch, or sinus, which had hitherto escaped my observation, and which extended backwards for nearly an inch by the outer side of the lower jaw-bone. As this sinus was probably lined by the same indolent substance as the fistula which had already been obliterated, I proposed to cauterize its interior, and on the 14th of December, an attempt was made to accomplish this, by introducing into it a piece of platinum wire doubled back upon itself so as to form a close bend, along which the electric current was made to 
pass, its two ends being previously connected with the two poles of the battery, which were carried into the mouth. The operation was continued for ten seconds only, but it did not prove successful; and accordingly, on the 18th of December, I repeated it in the same manner, the electric current being allowed to flow for fifteen seconds. The wisdom tooth, which rather crowded the jaw, was also extracted by my friend Mr. Samuel Morris, who assisted me at the time. The cautery produced rather more pain than in the first operation, a very thick slough was thrown out, and the sinus finally closed in somewhat less than a fortnight. During the progress of the cure, and for some weeks subsequently, good diet, wine, and tonics were prescribed. Up to the present date, March 22d, 1851, the cheek has continued quite sound, and the deep puckering of its outer surface is fast disappearing.

The case just related satisfactorily illustrates the peculiar advantages of the method of cauterization by means of the heat of electricity. The electric cautery, as it may conveniently be called, has the recommendation of being under proper care, intense, rapid, certain and uniform. It is simple and easy of application; for the wire, or other substance to be heated, may be duly and deliberately adjusted, whilst yet unconnected with the source of heat : its employment is unaccompanied by the terrors of a formidable apparatus, as the battery may be placed in an adjoining room : it may be used in deep-seated parts, and under many conditions in which no other cautery could be applied: its effects may be carefully measured, and are completely under control, being immediately arrested by breaking the galvanic current: it is so limited in its action as to cause no unnecessary destruction, or injury to adjacent parts ; and lastly, its use appears to be safe, and its consequences favorable.

It may here be mentioned, that subsequently to the cure. of the above-mentioned fistula, my attention was called to the fact that M. Fabre Palaprat had already used a platinum wire heated by galvanism for producing deep-seated moxas. 
His method is described, though not very intelligibly, in Becquerel's 'Traité de l'Electricité,' 1836, vol. iv, p. 306, but it is not stated that M. Palaprat employed it, or even recommended it, as a curative agent in the treatment of surgical disease.

For all such cases, however, in which potential cauterization may be advisable, and in which neither the common heated wire nor button is available, the electric cautery will, I feel assured, be employed with advantage, as, for instance, in obstinate fistulæ, leading into the mouth, or communicating with the trachea; in intractable perineo-urethral or rectourethral fistulæ; for cauterizing the edges of vesico-vaginal fistulæ, or the internal surfaces of long sinuses, resulting from abscesses, or from incompletely obliterated cysts; for arresting hæmorrhage in certain parts, otherwise difficult of access ; for uniformly cauterizing deep poisoned wounds, and in other conceivable instances.

But during the progress of my experiments, another and very different application of the electric cautery suggested itself, viz., that of employing the incandescent wire in the section of soft parts in the living body. In the experiments on dead animal tissues, I had found that a considerable thickness of muscular substance could be cut through by the heated wire in a few seconds; and I had further ascertained, in the living animal, that no hæmorrhage occurred, from the division, by the hot wire, of veins as large as a crow-quill, or of arteries a very little smaller.

It seemed probable, therefore, that it would be simple and safe to apply this method for the removal of redundant vascular parts, instead of the knife, scissors, or ligature; as, for example, in hæmorrhoids, both external and internal; polypi of the uterus ; and certain forms of erectile tumours, or any other soft pedunculated growths. For extirpation of diseased portions of the tongue or of the os uteri, it also appeared likely to be useful. Moreover, in certain peculiar cases, where simple division of the tissues alone was required, it seemed worthy of trial, as in the various kinds of rectal fistulæ, or in fissure of the anus. As a means of obliterating 
varicose veins, it might also prove safe and efficacious. Lastly, in the case of persons having a strong hæmorrhagic tendency, this mode of dividing the soft parts appeared to offer great advantages.

It was evident that the application of the actual cautery to such purposes, would be open to the objection of returning to a means of cure already abandoned as barbarous; but the peculiarities of this method of cauterization appeared to give such a command over the agent to be employed, as had been hitherto impracticable. I have accordingly used it in four cases of rectal fistulæ; in five cases of internal hæmorrhoids; in two cases of external hæmorrhoids, and in one of fissure of the anus. As some of these cases are still under treatment, and as their number is yet too inconsiderable for the purpose of fully testing a method so entirely new and open to improvement, I propose to defer, till another occasion, a statement of the results of these and of such other operations as I may hereafter have the opportunity of performing. In the meantime, I may state, that no bad consequences have followed in any case, and that, at present, all have been attended with success.

\section{Description of the apparatus employed, and of the mode of operating.}

The apparatus necessary for operations by the Electric Cautery consists of a moderately powerful galvanic battery, fitted up with flexible poles, one of which is interrupted at a mercury cup ; of terminal copper holders for the cauterizing wire ; and of platina wires of various thickness, fitted up with plates or balls of the same metal, if required.

The kind of battery is quite indifferent ; but I have used, on the ground of economy and simplicity of arrangement, one composed of iron and zinc on Sturgeon's principle, which, as a long-continued current is never needed, answers perfectly. Each cast-iron cell has about 120 square inches of surface, whilst each zinc element has 96. Four, six, eight, or nine such cells are used, according to the length of wire required to be heated. The nine cells will heat 4 inches 
of platina wire $\frac{1}{30}$ th of an inch thick in two seconds, and 6 inches in four seconds. The acid employed is one part of sulphuric acid to ten of water. This battery being, however, objectionable, from its size and weight, from the necessity of fixing the elements every time it is used, and from the large quantity of fluid employed, I am now having constructed one of a different form, which will be more compact and lighter, having the elements permanently fitted, and requiring, moreover, very little acid to work it.

The poles, which are necessarily stout, and which, therefore, if composed entirely of copper, would be very stubborn, may be made flexible by having a part of each composed of a column of mercury enclosed hermetically in a vulcanised indian-rubber tube, into each end of which the copper part of the pole is to be fitted. Moreover, one pole should be interrupted at a mercury cup, so as to render it easy to close and open the circuit instantaneously.

To the ends of the poles, stout copper holders, which may be plated, are fitted or unfitted at pleasure; these are constructed with a slit, to receive the cauterizing wire, which is fixed during the operation, and held tight by a sliding ferrule or screw.

Method.-In operating on a fistula, open at each end, the platina wire is first passed through the fistulous passage; its ends are then fitted on to the holders and these on to the two poles. The soft parts around being held aside, and the wire carefully disposed, the circuit is closed at the mercury cup, for a period calculated beforehand, as necessary to complete the cauterization.

If the passage be a cul-de-sac, the wire may, if practicable, be carried through it on a nævus-needle, and then be used as just now described; or, being first fixed to the holders, it may be bent into a loop, (having its two sides kept apart,) which is then inserted into the sinus, and the electric current made to pass along it.

The length of wire between the poles should just correspond with that of the fistula or sinus to be cauterized; and the time necessary for the operation may be best determined by 
allowing about twice the period taken by the same wire and battery to cauterize a track of the same length through a piece of dead muscular tissue.

When soft parts are to be quickly divided by the electric cautery, as in the case of fistula in ano, the wire should be at least twice as long as the thickness of the part to be cut through, so that it may be drawn, during the operation, to and fro with a sawing movement.

For the removal of soft parts, the wire (being fixed to the holders in the form of a loop, twice as long as the thickness of the part to be cut away,) is placed against the base of that part, and being heated, is used with or without a slight sawing movement.

If the part to be removed be large, it may be first transfixed by the wire, which being then properly fitted to the poles of the battery and heated, is made to divide one half of the mass, and then, without being unfitted, is carried to the bottom of the cut already made and drawn through the remaining half.

In proportion to the vascularity of the part to be divided or removed, the wire must be thicker, the heat less intense, and the sawing more gently performed, so that the section may be gradual and the cautery be allowed a longer time to penetrate and close the larger vessels.

Lastly, other modes of application, by varying the forms of the platina employed, will be suggested according to circumstances. 